\title{
Lebenskompetenz entwickeln im Dialog - Zur Aktualität der Pädagogik Friedrich Fröbels
}

\author{
Karl Neumann
}

\begin{abstract}
Zusammenfassung
Fragt man im Jahr des 175. Jubiläums der Gründung des Kindergartens nach der spezifischen Aktualität des Erbes von Werk und Person Friedrich Fröbels, zeigt sich bei Fröbel, auch im Vergleich mit den anderen „Klassikern“ der (Früh-)Pädagogik, nicht nur eine unmittelbare Anschluss-, sondern sogar eine besondere Zukunftsfähigkeit im Hinblick auf die gegenwärtigen Forschungs- und Diskussionsstränge der Frühpädagogik. Fröbels Kindheitspädagogik ist in ihrem Kern kommunikativ-dialogisch konzipiert. Sie hält insofern nach wie vor grundlegende Anregungen bereit zu den Themen phänomenographische Erfassung der Bildungsprozesse und deren curriculare Gestaltung; Kooperation zwischen Eltern und Erzieherinnen; Professionalisierung des Personals, schließlich hinsichtlich der kinderrechtlichen und bildungspolitischen sowie ökonomischen Herausforderungen und Konsequenzen. In kontrastivem Abgleich mit dem derzeit in den Bildungswissenschaften dominierenden Begründungsdiskurs der Bildungsstandards-Paradigmatik wird auf das kritische Potential der Fröbelschen Bildungstheorie verwiesen und für das Feld der Didaktik der Frühpädagogik am Beispiel der Spielpädagogik Fröbels expliziert. Für Fröbel steht dabei, wie in jüngster Zeit insbesondere in der Sozialpädagogik thematisiert, der Erwerb von Lebenskompetenz im Zentrum. Dabei besteht allerdings bei Fröbel kein Zweifel, dass eine didaktisch arrangierte Auseinandersetzung mit Welt und Selbst als Begreifen und Gestalten des eigenen Lebens nur als Prozess einer religiösen Selbstverständigung modelliert werden kann. Mit seinem Konzept vom Kindergarten als einem die ,sphärische“ Grundverfasstheit der Welt repräsentierenden Ort hat er offenbar ein kontinuierlich weiterwirkendes Modell des Lebensortes für Kinder in ihrer sie jeweils umgebenden Gesellschaft nicht nur begründet, sondern auch schon experimentell erfolgreich realisiert.
\end{abstract}

Schlagworte: Aktualität des Klassikers Fröbel, Didaktik der Frühpädagogik, Spielpädagogik, Bildungstheorie und Bildungsstandard-Diskurs, Lebenskompetenz, Kindergarten-Idee als konkrete Utopie

Developing life skills in dialogue - About the actuality of Friedrich Froebel's pedagogy

\begin{abstract}
Wondering about the specific actuality of the heritage of the work and the person of Friedrich Froebel in the year of the 175th anniversary of the foundation of the kindergarten, it can be seen that Froebel's work, in comparison to other "classics" of (early childhood) education, is not only compatible to today's research and discussions in the field of early childhood education, but is furthermore relevant for the future. The concept of Froebel's early childhood education is basically communicative-dialogic. Furthermore, it still provides essential stimuli on topics such as the phenomenographic research on educational processes and their curricular design; the cooperation of parents and educational staff, the professionalization of the staff and the challenges concerning children's rights, educational policy and economic consequences. In contrast to the currently dominating foundational discourse of educational standards, the critical potential of Froebel's educational theory is highlighted. His theory is explicated for the field of the didactics of early childhood education using the example of Froebel's pedagogy of play. Froebel focuses on the acquisition of life
\end{abstract}


skills similar to the current discourse in the field of social education. However, there is no doubt that the didactically arranged examination of the world and the self as an understanding and a design of the own life according to Froebel - can only be seen as a process of a religious self-understanding. With his concept of the kindergarten as a place representing the ,spherical” basic structure of the world, Froebel did not only found but also successfully realized an ongoing setting for children in society.

Keywords: Actuality of Froebel's pedagogy, didactics of early childhood education, pedagogy of play, educational theory and educational standards, life skills, idea of kindergarten as a specific utopia

\section{Der Kindergarten als Lebensraum - Zur Wirkungsgeschichte der Idee des Kinder-Gartens als konkrete Utopie}

In einer Beilage der ZEIT, Reihe ,Geschichte“, vom November 2009, die „Vordenker, Vorbilder, Visionäre - 50 Deutsche von gestern für die Welt von morgen“" porträtierte, findet sich auch Friedrich Fröbel einbezogen in diesen Kreis von „Menschen der Vergangenheit, die man nicht nur ihres Werkes wegen, sondern auch als Charaktere verehrt", einen Kreis, der ausdrücklich „keinen Katalog aus dem historischen Persönlichkeitsbaumarkt, sondern ein lebendiges Bild der Gegenwart“ und zugleich „der Wünsche an unsere Zukunft“ aufzeigen sollte (Erenz 2009, S. 3). Als faszinierende, zentrale Merkmale der „Zukunftsfähigkeit" Fröbels stellt Thomas Kerstan die Komponenten seiner Idee von Kindheit heraus: „Selbstbewusst und froh sollen die Kinder sein, im Spiel die Welt entdecken. Fröbels Konzepte inspirieren die Pädagogen bis heute“ (ebd., S. 15).

Als ein spontan assoziierter Beleg für die hier für Fröbel beanspruchte Zukunftsfähigkeit lässt sich das (immer wieder zitierte) quasi überhistorische Motto lesen, „Kommt, lasst uns unseren Kindern leben!“‘, mit dem Fröbel Konzeption und Einrichtung seines „Allgemeinen Deutschen Kindergartens“ (1840), getragen von der Begeisterung seines nimmermüden pädagogisch-politischen Engagements, in die breite zeitgenössische Öffentlichkeit, insbesondere die bürgerlichen Schichten, hineinzutragen versuchte (Rockstein 2015). In seinem Spätwerk hatte Fröbel seine Leitvorstellung von „Menschenerziehung“, nämlich „freie, denkende, selbstthätige Menschen zu bilden“ (Brief an Krause, 1828; Fröbel 1982a (Bd. 1), S. 149) weiterentwickelt zu einer ausdifferenzierten Pädagogik schon der kleinen Kinder als „Bildung von Anfang an“. Er knüpfte aber durchaus auch an eine der Grundüberzeugungen der Aufklärungspädagogik an, Kindheit immer auch als „Ressource“ zu betrachten, von deren planmäßiger und richtiger Nutzung wie in anderen Bereichen des staatlichen und ökonomischen Lebens das Wohl der (bürgerlichen) Gesellschaft entscheidend abhängig erschien (Neumann 1993, S. 192ff.). Als Schulpädagoge und Lehrerbildner hatte Fröbel genug praktische Erfahrung dazu gesammelt, dass es zur Realisierung seiner Idee vom Kind als eigenständigem Subjekt für die frühpädagogische Bildung einer institutionellen Rahmung bedürfte. „Kindergarten soll die Anstalt heißen", lautete entsprechend seine Folgerung.

Fröbels Konzept des Kindergartens begann noch zu seinen Lebzeiten, weltweite Verbreitung zu finden (vgl. Erning/Neumann/Reyer 1987). Bis heute „überstrahlt [...] das Wort ,Kindergarten“ als eines der schönsten und erfolgreichsten deutschen Exportwörter solche zweifelhaften Welterfolge wie ,Weltschmerz", ,Angst" und ,Blitzkrieg“,, (Kerstan 2009). Das zeittypische Institutionsformat „Anstalt“ deckt begrifflich allerdings weniger als die Hälfte der Bedeutung von Fröbels Idee des Kindergartens ab. Es dürfte das ambi- 
valent-utopische Anregungspotential sein, das Idee und Ort des „Kinder-Gartens“ von Beginn an implizierte, die dialektische Verknüpfung von „Anstalt“ und „Lebensraum“, die Fröbel mit seinem pädagogischen Prinzip einer lebensweltlichen Überwindung der Dissoziationen zwischen Erwachsenen und Kindern, zwischen Privatheit und Öffentlichkeit, zwischen familialem Haushalt und öffentlicher Veranstaltung im Kindergarten zu einer Einheit vermittelt sehen und gestalten wollte.

Insbesondere Jürgen Reyer hat seit den 1980er Jahren bis in die jüngste Zeit in einer Reihe von Studien zum Spannungsverhältnis zwischen „Ver- und Entanstaltung“ als Strukturelemente des Fröbelschen Kindergartenprinzips die Geschichte der unterschiedlichen Formate der Realisierung von „Kindergarten als Lebensraum“, national wie international, zu rekonstruieren versucht. Die bekannte Vielfalt an institutionellen Erscheinungsformen und Programmatiken in den vergangenen 175 Jahren, auf denen das Etikett ,Fröbel“" zwar aufgebracht, „Fröbel“" aber nicht - salopp gesprochen - „drin war", würde Fröbel selbst kaum glücklich gemacht haben (Reyer 1988, 2010). Die Intensität der Diskussion zum Prinzip „Kindergarten als Lebensraum“ in pädagogischer Theorie, Praxis oder Politik über mehr als ein Jahrhundert, auch wenn sein Name nicht immer Erwähnung fand, dürfte Fröbel, der so oft scheiterte mit der Umsetzung der eigenen Praxisprojekte, inzwischen wohl mit Befriedigung und Stolz erfüllen.

Beispielhaft dafür kann hier hingewiesen werden auf den Fachkongress „KINDHEIT '90“, organisiert unter der Federführung des Pestalozzi-Fröbel-Verbandes (PFV), der abschloss mit einem flammenden Plädoyer Jürgen Zimmers für ,eine Politik für Kinder als konkrete Utopie“, der radikalen Forderung, „Pädagogik am Leben neu zu vermessen“ (Zimmer 1991, S. 231). Sigrid Ebert, die damalige Vorsitzende des PFV, erinnerte ausdrücklich an die damals 150 Jahre zurückliegende Gründung des Kindergartens durch Fröbel als dessen „Antwort“ auf „die zur damaligen Zeit sich vollziehenden spezifischen Veränderungen in der Lebenssituation von Familien mit Kindern“" (Ebert 1991, S. 13).

Die nicht zuletzt mit Fröbels Idee vom Kindergarten angestoßene und seitdem nicht zur Ruhe gekommene Diskussion zu den Fakten und Konsequenzen der im Prozess der Modernisierung fortschreitenden Aufspaltung des kindlichen Lebens-, Erfahrungs- und Erziehungszusammenhangs hat, ohne durchgängig einen direkten Bezug zu Fröbel herzustellen, im Tagesbetreuungsausbaugesetz der BRD (2005) einen vorläufigen Ankerpunkt gefunden, und zwar mit dem Leitbild der Erziehungs- und Bildungspartnerschaft im Modell eines kooperativen Projekts aller am Erziehungsprozess Beteiligten. Aufgenommen sind hier u.a. Leitvorstellungen des 10. Kinder- und Jugendberichtes, zum Wohl der Kinder eine „Kultur des Aufwachsens“ zu sichern, d.h. „Kooperationsformen, in denen Eltern und andere Erzieher sich in ihren differenzierten Rollen gegenüber dem Kind gegenseitig stützen, so dass Kinder nicht insulare Erfahrungswelten durchwandern, in denen kein übergreifender Sinn gilt" (BMFSFJ 1998, S. 19). Eine Art vorläufige Zwischenbilanz der bildungstheoretisch-pädagogischen wie der bildungspolitischen Diskussion in Theorie und Praxis ist mit dem 12. Kinder- und Jugendbericht gezogen worden: „Das angesammelte Wissen über Bedingungen, Beeinträchtigungen und Risiken der Entwicklung von kleinen Kindern macht einen Dialog und eine gemeinsam geteilte Verantwortung für das Aufwachsen der Kinder erforderlich. [...] Da die Entwicklungs- und Bildungsprozesse der Kinder weitgehend von den Umwelten abhängig sind, in die sie hineinwachsen [...], ergibt sich als große gesellschaftliche Herausforderung, die Qualität der Bildungswelt der Familie ebenso wie die der Bildungsorte Tagespflege und Kindertageseinrichtungen sowie sonstiger bildungsrelevanter Erfahrungsräume und Lernwelten zu schaffen. Es liegt 
im öffentlichen Interesse, dass die Kinder sich gesund und ihre Möglichkeiten ausschöpfend entwickeln können, damit sie an der Gesellschaft umfassend teilhaben können, die ihrer bedarf" (BMFSFJ 2005, S. 130).

Erst seit 1996 hat in der BRD jedes Kind das Recht auf einen Kindergartenplatz. Die Ausbau- und Besuchsstatistiken zum Kindertagesstättenbereich und zur Tagespflege seit diesem Datum demonstrieren, wie selbstverständlich inzwischen die Inanspruchnahme dieser Institutionen geworden ist. Die Radikalität der kinderpolitischen Wende, wie sie schließlich in der gesetzlichen Wende des Rechtsanspruchs auf einen Kindergartenplatz zum Ausdruck kam, wird jedoch erst in der historischen Perspektive angemessen einschätzbar. Ludwig Liegle hat in einem prägnanten Rückblick auf die Stationen der Entwicklung der Kindergartenidee herausgestellt, welches transformatorische Potential „Fröbels Vision eines allgemeinen Kindergartens - als erste Stufe des deutschen Bildungswesens und ,Vermittlungsinstanz ${ }^{6}$ zwischen Familienerziehung und Schulunterricht - sowie die Umsetzung dieser Vision durch Kindergärten [...] und Ausbildungsstätten für Erzieherinnen“ aufwies, das Fröbel „zum Vater der Idee eines pädagogisch und sozialpolitisch begründeten Anspruchs jedes Kindes auf familienergänzende und -unterstützende Erziehung, Betreuung und Bildung werden“ ließ (Liegle 2013, S. 29).

Liegle prüft in diesem Kontext auch die These, inwieweit Fröbels Idee vom autonomen Kind und dem Kindergarten als gesellschaftlich-öffentlicher Institution auch den entscheidenden Anstoß für die weltweite Kinderrechtsbewegung lieferte, insbesondere für die Verbreitung der kindlichen Schutz- und Förderrechte. Den wichtigsten Beleg dafür bildet aus seiner Sicht die Nachwirkung der Fröbelschen Programmatik in der internationalen Fröbelbewegung (vgl. Surall 2005). Sie setzt im 19. Jahrhundert ein und hält bis in die Gegenwart an (vgl. Heiland/Neumann 1998, 2003; Heiland/Neumann/Gebel 1999). Einen sprechenden Beleg dafür bildet für Liegle die weltweit erste Buchveröffentlichung über Kinderrechte aus der Feder einer Fröbelianerin. Kate Douglas Wiggins Buch „Children's Rights. A book of nursery logic“ (1892) „stützt sich weithin auf Fröbels Ideen und seine didaktischen Materialien (Spielgaben), setzt aber auch einen eigenen Akzent, indem Wiggin den Beitrag des Kindergartens zur Sozialreform herausarbeitet" (Liegle 2013, S. 30). Wiggins Schrift „Kindergarten principles and practice“ (1886) erschien im Jahre 2009 in einem Reprint.

Erst eine historische Analyse von Programm und Person Friedrich Fröbels vermag den Weg zum Facettenreichtum ihrer Aktualität in voller Breite zu eröffnen, ein typisches Beispiel für die gleichzeitige Zeit- wie Unzeitgemäßheit eines sog. pädagogischen Klassikers. Fröbels Vision vom Kindergarten war angesichts der gesellschaftlich-politischen Verhältnisse in seiner Zeit eine reformatorische bis revolutionäre Herausforderung und ist dies bis heute geblieben (vgl. Winkler 2010).

\section{Lebenskompetenz entwickeln im Spiel und durch Spielpflege - Fröbels Impulse für die aktuellen Ansätze dialogorientierter Frühpädagogik}

Die folgenden Überlegungen gehen von der Grundannahme aus, dass Fröbels in seinem Spätwerk konzipierte Kindergartenpädagogik in ihrem Kern kommunikativ-dialogisch strukturiert ist und insofern grundlegende Anregungen bereit hält für die aktuelle konzep- 
tionelle Debatte in der Frühpädagogik zu den Themen phänomenographische Erfassung der Bildungsprozesse und deren curriculare Gestaltung; Kooperation zwischen Eltern und ErzieherInnen (einschließlich konkreter Maßnahmen zur Familien-, insbesondere Mütterfortbildung oder moderner Feedback-Verfahren, wie Beobachtungsprotokolle und Lernberichte, z.B. durch Briefe an die Eltern); Professionalisierung des Personals, schließlich, wie bereits aufgezeigt, hinsichtlich der kinderrechtlichen und bildungspolitischen sowie ökonomischen Herausforderungen und Konsequenzen. Aus der Fülle der genannten Aspekte, die sämtlich unterschiedlich breit und tief in der deutschen oder internationalen einschlägigen Theorie und Praxis nach wie vor aufgegriffen werden, werde ich mich hier auf den wohl wichtigsten konzentrieren, die konzeptionelle Modellierung des kindlichen Bildungsprozesses und dessen curriculare Gestaltung, kurz gefasst: Fröbel und die frühpädagogische Didaktik. Von ähnlichem wirkungsgeschichtlichen Gewicht wären Fröbels Beiträge zur Professionalisierung des früh- bzw. sozialpädagogischen Personals (vgl. dazu Sigrid Eberts nachfolgenden Beitrag in diesem Heft).

\subsection{Fröbels frühpädagogisches Didaktik-Konzept vor dem Hintergrund der aktuellen Debatte über Bildungsstandards}

Der Zeitgeist ist für eine Rezeption der frühpädagogischen Klassiker wenig günstig, ganz zu schweigen von einem expliziten Rückgriff auf die authentischen Texte (vgl. Neumann 2013b). Denn didaktische Problemstellungen sind heute, auch in Theorie und Praxis der Frühpädagogik in zunehmendem Maße, auf den Nachweis messbarer Leistungen als Resultat von Lernprozessen in verschiedenen Domänen von Kompetenzen ausgerichtet. Allerdings hält die Tradition der Klassiker, und hier insbesondere Fröbel, ein Anregungspotential bereit, das integrativ in die Lernbereiche der verschiedenen, auf Standards ausgerichteten Bildungspläne einbezogen werden kann, die derzeit in allen deutschen Bundesländern vorgelegt bzw. umgesetzt werden. Fröbel-Spielpflege im Kindergarten, beispielsweise als Zusammenwirken von Bewegungsspiel (Sprache/Sozialverhalten), Spielpflege mit Materialien (Mathematik/Sachkunde) und Gartenpflege (Natur/Ökologie), zielt auf den Erwerb elementarer Kompetenzen. Dabei sieht Fröbel den Erwerb von Handlungskompetenz unmittelbar verbunden mit in Stufen wachsender, bewusster Einsicht, also metakognitiven Lernprozessen, ausgehend von zunächst ,ahnendem“ Erfassen, weitergeführt zum ausdifferenzierten Begreifen der Strukturen und Gesetzmäßigkeiten der Welt der Dinge und Personen bis hin zum Selbstkonzept einer kategorialen Bildung.

In der frühpädagogischen Grundlagendebatte ist, nach der Einführung von standardorientierten staatlichen Rahmenrichtlinien für die pädagogische Arbeit in Kindertageseinrichtungen, national eher in Bildungsplänen, international in Curricula (vgl. Fthenakis 2003), auch in Deutschland die Suche nach einer Didaktik der Frühpädagogik erheblich belebt worden, ohne dass inzwischen ein allgemein akzeptierter theoretischer Bezugsrahmen didaktischer Modellkonstruktion vorliegen würde. Diese Debatte bezieht auch die historische Dimension mit ein.

In dem von Dagmar Kasüschke herausgegebenen Übersichtsband „Didaktik in der Pädagogik der frühen Kindheit" (2010) hat Helmut Heiland, gleichsam als Beginn einer weiter auszuziehenden Traditionslinie, die auf Fröbel zurückgehende Programmatik der bildungstheoretischen Modellkonstruktion in ihrer aktuellen Bedeutung am Beispiel der Fröbelschen Spielpädagogik herausgestellt. Er zeigt, wie Fröbel zentrale Bausteine für 
die didaktisch-methodischen Dimensionen der Frühpädagogik zusammengefügt, jedoch nicht mehr expressis verbis zu einer konsistenten Theorie ausformuliert hat (Heiland 2010, S. 17ff.). In diesem Kontext muss allerdings sogleich daran erinnert werden, dass der „Vater" des aktuell in der BRD nach wie vor einflussreichsten Didaktik-Modells, der sog. bildungstheoretischen Didaktik, Wolfgang Klafki (vgl. Wigger 2010, S. 276ff.), die grundlegende konzeptionelle Anregung für sein Konstrukt von „Bildung“ als didaktischer Zentralkategorie nicht zuletzt im Anschluss an Fröbels Modell von Bildung gewonnen hat: „Die bildende Wirkung der am Konkreten gewonnenen allgemeinen Inhalte ist eben die, daß sie nicht äußere Maßstäbe bleiben, die an die ,Welt' angelegt werden, sondern daß sie - als Grundformen des Anschauens, Denkens, Erlebens, Wertens - Wesensmomente des Gebildeten selbst werden, ,Kategorien“ künftiger Begegnung des Menschen mit der ,Welt' und sich selbst. Es waltet hier ein unauflösliches Wechselverhältnis: Das BildendAllgemeine ist das Allgemeine des Konkreten, ist nur in ihm und um seiner willen; aber das Konkrete als Begriffenes, Verstandenes, Erlebtes, Geleistetes im Sinne der Bildung ist umgekehrt immer das Konkrete eines Allgemeinen“"(Klafki 1963, S. 35). Von Klafkis Position ließe sich durchaus eine Linie der Argumentation zu einem zeitnahen Modellierungsversuch weiterführen, wie er von Susanne Viernickel und Ursula Stenger (2010), ausdrücklich als Vorarbeit gekennzeichnet, unter dem Titel „Didaktische Schlüssel in der Arbeit mit null- bis dreijährigen Kindern" im erwähnten Sammelband vorgelegt worden ist (ebd., S. 175ff.) - ohne dass dabei Klafki oder Fröbel Erwähnung fänden.

„Bildung“" wird von den Autorinnen als eine „besondere Form des Lernens“ verstanden, insofern sie „den subjektiven Sinn und die Bedeutsamkeit“ berücksichtige, „die erworbenes Wissen und Können bzw. erlerntes und angewendetes Verhalten für das Individuum besitzt. Unter Bildung sind dann nur diejenigen Anteile von Entwicklungs- und Lernprozessen zu verstehen, die dazu beitragen, dass das Individuum seine Möglichkeiten des Handelns, Fühlens und Denkens gegenüber sich selbst sowie der sozialen und materiellen Umwelt erweitert“, damit auch die Möglichkeiten verstärkt, „selbstwirksam agieren zu können“. Der Bildungsprozess wird hier - und das ist ein fundamentaler Unterschied zu Klafki - nicht primär von den Bildungsinhalten, sondern den psychisch-physischen Vorgaben des Lernvermögens, also den kognitiven, motorischen, motivationalen und volitionalen Dimensionen an Kompetenzen zum Problemlösen, didaktisch modelliert. Viernickel und Stenger unterscheiden dabei die Dimensionen 1. der Beziehungsbildung, 2. die Ausbildung der Fähigkeiten der aktiven Aneignung der Welt (also die Handlungsdimension), 3. die Aneignungs- und Ausdruckstätigkeit und schließlich 4. die Persönlichkeitsoder Identitätsbildung (ebd., S. 177).

Entlang dieser Dimensionen als Basis - also im paradigmatischen Einklang mit den derzeit dominierenden bildungswissenschaftlichen Forschungsstrategien - ließen sich prinzipiell inhaltlich strukturierte Standardkataloge und damit Ebenen für ausdifferenzierte Kompetenzhierarchien mit jeweils zugeordneten Itemclustern für empirisch kontrollierbare Wirkungs- bzw. Outcome-Analysen konstruieren (Klieme u.a. 2003; Köller 2008). Auf diesem Weg, der nach den internationalen Bildungsvergleichsstudien vor allem im Hinblick auf die Entwicklung schulischer nationaler Bildungsstandards oder den Europäischen Qualifikationsrahmen kontinuierlich ausgebaut wurde, wird der Bildungsbegriff lediglich ansatzweise in didaktisch-erziehungswissenschaftlichen oder psychologischen Modellen elaboriert. Was unter „Bildung“ zu verstehen ist, wird weniger theoretisch abgeleitet als konsensuell-kumulativ festgelegt. Der wichtigste Orientierungsrahmen für einen solchen Konsens über die notwendigen Inhalte von Bildung wird damit nach 
wie vor in hohem Maße traditionell-domänspezifisch aus den in (Schul-)Fächern, ihren Bildungsplänen und Curricula vorliegenden Fachinhalten geliefert.

Fröbel dagegen hat im Konzept seiner „Menschenerziehung“ bzw. „Spielpflege“ ein theoretisch modelliertes Leitziel für Bildung, und zwar abgeleitet als pädagogische Norm aus seiner Bildungsphilosophie und Anthropologie. Das Ziel der Erziehung des Kindergartens ist es, das Kind in seinem Entwicklungsprozess die Einigkeit mit sich selbst, mit der Natur, mit der Menschheit und mit Gott finden zu lassen. In seiner Rekonstruktion der didaktischen Struktur der Fröbelschen Spielpflege weist Sigurd Hebenstreit diesem Einheits- bzw. Identitätsprinzip den Leitzielcharakter für den Rahmen der übrigen Bildungskategorien zu. Fröbels primäre Intention sei die auf „Einigung“, „Einigkeit“ gerichtete „Vermittlung“, die Bestätigung der „Ahnung“ des Kindes, dass, in Fröbels Worten, ,aller Mannigfaltigkeit eine Einheit, allem Sichtbaren ein Unsichtbares, allem Äußeren ein Inneres, allem Unbelebten ein Belebtes und zuletzt die höchste Einheit zum Grunde liegt, die wir als gut an sich erkennen und darum Gott nennen“" (Hebenstreit 2003, S. 392ff.). Konsequenterweise beanspruchte Fröbel dann Termini wie „Einigkeit und Mannigfaltigkeit“, „Gegensätzlichkeit“, „Allseitigkeit“ oder „Allharmonie“" als grundlegende bildungstheoretische Kategorien. Sie erscheinen angesichts der gegenwärtig in den Bildungswissenschaften gebräuchlichen wie aus der Zeit gefallen.

Kann man deswegen schon, quasi als längst „überholt“, Fröbels Konzept gleich „vergessen"? Aus der aufgezeigten paradigmatischen Kluft der didaktischen Modelle resultiert vielmehr die Frage, ob man nicht in der Auseinandersetzung gerade mit Fröbel (dessen anthropologisch religiös-metaphysisch begründetes Bild vom Menschen man vielleicht überhaupt nicht akzeptiert) auf den springenden Punkt der aktuellen DidaktikDebatte, den nach wie vor kontrovers diskutierten Bildungsbegriff, gestoßen werden kann. In einer eindringlichen Studie „Menschenerziehung - Lernen das Leben zu lesen“ hat sich Michael Winkler (2009) mit zwei die bildungswissenschaftlich wie die bildungspolitische Debatte dominierenden Grundkonzepten, nämlich Kompetenz und Literacy, sehr kritisch hinsichtlich ihrer begrifflichen Konsistenz auseinandergesetzt. Beide Konzepte erfassten eben nicht, was sie als Ziel ausgeben: Die Welt in ihren Strukturen zu beschreiben und zu erklären, z.B. durch frühen Anschluss der Kinder an wissenschaftliches Wissen. Sie unterschätzten systematisch, im Gegensatz zu Fröbels Modellierung pädagogischer Prozesse, die Komplexität des Vorgangs, die Thema sein muss, „wenn wir lernen müssen, das Leben zu lesen“" (ebd., S. 65).

Fröbels Didaktik-Konzept erscheint, schwerpunktmäßig im Bereich der Frühpädagogik, insofern besonders geeignet, Chancen und Risiken der seit den weltweiten Bildungsvergleichsstudien dominierenden „Instandardsetzung“ des Bildungswesens (Schlömerkemper 2004) im Hinblick auf intendierte und nicht-intendierte Effekte der laufenden Reformprojekte kritisch auszuloten. Dabei dürfte die Debatte über „Bildungsstandards außerhalb der Kernfächer" (Tenorth 2008) und dabei wiederum die Rolle von Bildungsstandards für den Religionsunterricht bzw. die religiöse Bildung von besonderem Interesse sein (vgl. Krause/Nikolova/Schluß/Weiß/Willems 2008; Rothgangel 2008). Es muss durchaus als Manko der Fröbel-Forschung betrachtet werden, dass bisher eine empirisch belastbare Wirkungs- oder Evaluationsstudie zum Einsatz von Fröbels Spielgaben, etwa im Vergleich zum Stand mathematischer Frühförderung im Kindergartenalter (vgl. z.B. Meyerhöfer 2010), immer noch nicht vorliegt. Für den systematisch-theoretischen Begründungsdiskurs im Kontext der Bildungsstandard-Paradigmatik hat, angesichts seiner grundlegenden konstitutiven Bedeutung für Fröbels Didaktik, allerdings die prinzipielle 
Berücksichtigung des Bereiches „religiös-konstitutive Rationalität“ neben „kognitiver, moralisch-evaluativer und ästhetisch-expressiver Rationalität" vorrangiges Gewicht. Diese ist in der PISA-Studie 2000 zwar erwähnt, bekanntlich aber nicht eigens untersucht und wird erst allmählich für die hinter den Bildungsstandards liegenden Kompetenzmodelle theoretisch elaboriert (vgl. Deutsches PISA-Konsortium 2001, S. 21).

Als springender Punkt der aktuellen frühpädagogischen Didaktik-Debatte kristallisiert sich immer mehr die Frage heraus, inwieweit die spezifische Logik (früh-)kindlicher Bildungsprozesse sozusagen nahtlos mit den Varianten der gegenwärtig dominierenden kognitions- und sozialpsychologischen lerntheoretischen Modelle adäquat erfassbar ist. Denn insbesondere didaktisch strukturierte, institutionalisierte Instruktionsprozesse bergen die Gefahr einer lernzielorientierten Instrumentalisierung des Lernpotentials im kindlichen Selbstbildungspotential. „Bildungsprozesse gehen zuvörderst von der Autonomie des Kindes aus, Instruktionsprozesse von den Lern- und Kompetenzzielen, die erreicht und didaktisch garantiert werden sollen. Bei Bildungsprozessen müssen in erster Linie die Bildungsziele der Kinder in Einklang mit den Möglichkeiten und Initiativen der Kinder gebracht werden. Bei Instruktionsprozessen müssen vor allem die Kinder in Einklang mit den vorgegebenen Zielen gebracht werden“ (Schäfer 2007, S. 57). Die gesellschaftlichpolitischen Leitvorstellungen gelingender Kindheit, wie sie exemplarisch in den staatlichen Rahmenvorgaben für das (vor-)schulische Bildungswesen zum Ausdruck kommen, sind schwerpunktmäßig auf das Kind als kompetenten Lerner ausgerichtet, der durch die Vermittlung grundlegender standardorientierter Kompetenzen und die Entwicklung wie Stärkung persönlicher Ressourcen motiviert und darauf vorbereitet werden soll, künftige Lebens- als Lernaufgaben zu bewältigen, verantwortungsvoll am gesellschaftlichen Leben teilzuhaben und ein Leben lang zu lernen (vgl. z.B. JMK/KMK 2004). Diese Leitvorstellungen können offensichtlich Gefahr laufen, das Bild vom Kind als kompetentem Lerner auf ein Bild vom Kind mit optimaler Lernausstattung zu reduzieren (vgl. Neumann 2013a, S. 20ff.).

Lernkompetenz ist jedoch eine gewiss notwendige, aber noch nicht die hinreichende Bedingung für die Lebenskompetenz von Kindern (vgl. Münchmeier/Otto/Rabe-Kleberg 2002). Kinder sehen ihre Welt durchaus als Lernwelt, allerdings immer auch aus der prüfenden Perspektive, ob die Bedingungen für ihr persönliches Wohlbefinden oder Glück, für eine Wertschätzung ihrer Weise der Welterfahrung und -mitgestaltung gegeben sind (vgl. Bucher/Münnich 2012). Die World Vision Kinderstudien (2007, 2010) haben das Bedingungsgefüge aufgeschlüsselt, in dem sich Kinder als Subjekte aus eigenem Recht mit ihrem Selbstpotential entfalten können (Andresen 2012).

\subsection{Fröbels Spielpädagogik als frühpädagogisches Konzept kategorialer Bildung}

In einer inzwischen langen Forschungstradition der Klärung des Bedingungsrahmens gelingender Kindheit ist der dialogische Prozess der Bindung zwischen Kindern und den sie betreuenden Erwachsenen als Bildungsbeziehung ausführlich analysiert worden (vgl. Drieschner 2011). Bindungs- wie Explorationsbedürfnisse des Kindes werden in einem festen Zusammenhang gesehen (vgl. Ahnert 2010, S. 47). Selbstbildung geschieht als eigenaktive Weltaneignung in sozialen Bezügen. Damit die Welterfahrungen kleiner Kinder „,bewusst werden und denkend genutzt werden können, braucht das Kind Menschen, die 
auf seine Erfahrungen eingehen, sie auf unterschiedliche Weise spiegeln und sie schließlich in Worte fassen" (Schäfer 2008, S. 8). Vom Forschungsteam um Michael Tomasello im Max-Planck-Institut für evolutionäre Anthropologie (Leipzig) konnte nachgewiesen werden, dass Kinder das stammesgeschichtliche Erbe einer ,geteilten Aufmerksamkeit“ bzw. „Wir-Intentionalität" als Basis kommunikativen Handelns und damit humaner Lebensbewältigung sozusagen „mitbringen“ (vgl. Tomasello 2011). Wir-Intentionalität als Basis dialogisch konstituierter, kultureller Aktivität offenbart sich in der frühen Kindheit vorrangig im Spiel. Das Spiel der Kinder ist, spätestens seit Friedrich Fröbel, als Protokoll der Weltaneignung gelesen, in pädagogischen Prozessen dialogisch strukturierter Erziehung begleitet und - insbesondere durch professionelle Spielfeinfühligkeit - gezielt unterstützt worden (vgl. Heller 2013). Eine Didaktik der Frühpädagogik kann in diesem Sinne traditionelle wie zukunftsorientierte Leitlinien zu einer Theorie ,spielenden Lernens“ verknüpfen, wie sie beispielsweise von Pramling Samuelsson und Asplund Carlsson (2007) - u.a. mit eingehender Diskussion der spielpädagogischen Didaktik Fröbels in ihrem international weithin beachteten Buch vorgelegt worden ist. Das pädagogische Leitbild früher Kindheit heute ist also fokussiert auf das Kind als kompetenten Lerner, dies aber nicht vorrangig in der Perspektive der Prototypik schulischen Unterrichts, sondern immer auch in der der Entdeckung und Beschreibung der spielerischen, informellen und inzidentellen Lernprozesse, für die allerdings die pädagogisch-gesellschaftlichen Umwelten als Kontext indirekter Erziehung geplant und gestaltet werden müssen (vgl. Liegle 2012).

Grundlegende Einsichten der aktuellen psychologischen Forschung zu den frühkindlichen Bildungsprozessen, die die Bedeutung des spielerischen, nicht-strategischen, inzidentellen (d.h. zufälligen) Lernens im Prozess des gesamten Lebenslaufs, insbesondere aber für das frühe Kindesalter herausstellen (vgl. z.B. Hasselhorn 2005), finden sich bereits bei Fröbel, z.B. phänomenographisch meisterhaft erfasst in seiner Analyse des Spiels im Kindergarten als „freie Beschäftigung“ zur Lebenserfahrung. Implizit liefert Fröbel dabei auch die Begründung dafür, warum „Spielpflege“ und „Unterricht“ im Kontext direkter oder indirekter Erziehung, z.B. in den Institutionen „Kindergarten“ und „Schule“, sorgfältig unterschieden werden sollten:

„In dem Kindergarten herrscht die freie Beschäftigung, das Spiel, wie der eigentliche Unterricht der Schule angehört. Da aber alle Beschäftigungen und alles Spiel schon durch sich unterrichtet, belehrt, mindestens die verschiedenen Seiten des Geistes entwickelt, bei der gewöhnlichen Art des Spielens (aber) diese unmittelbare, darum lebenvolle Sachbelehrung und Gegenstands-Unterricht nicht hervorgehoben wird, also unbeachtet bleibt, dieses Belehrende und Unterrichtende hier aber unmittelbar, wenn auch im Spiel und auf Spielweise hervorgehoben und beachtet wird, so sind diese Spiele unmittelbar durch sich selbst belehrend, sowohl im Allgemeinen als im Besonderen. [...] Dieses Spiel ist darum keineswegs ein nichtiges, gehalt- und fruchtloses Zeitvertreiben, ist nicht ein zufälliges ungeordnetes Leben und Bewegen, ist keineswegs ein Nichtslernen, sondern vielmehr ein ununterbrochenes Lernen, aber am, um, im Leben selbst. Das Gelernte geht hier sogleich wieder ins Leben über, ohne Gegenstand an sich zu werden, dadurch unterscheidet sich dieses Lernen von dem in der Schule, zu welchem das Kind dadurch erst vorbereitet werden soll." (Brief an T. Brunszvik, 1842; Fröbel 1982a (Bd. 3), S. 228)

Mit Fröbels konzeptionellen Argumenten im Hintergrund hat Liegle die „Perspektiven einer frühpädagogischen Didaktik der indirekten Erziehung“ (2012) systematisch weiterentwickelt. „Das Lernen der Kinder im Vorschulalter findet zu einem großen Teil unbewusst, beiläufig, zufällig statt. Dasjenige jedoch, was den Kindern als Gelegenheiten und Herausforderungen zum Lernen ,zufällt', hängt davon ab, was sie in ihrer Umwelt - in 
diesem Fall dem Kindergarten - vorfinden. Es kommt also darauf an - und damit sind wir bei der professionellen Verantwortung der Fachkräfte -, diejenigen Gelegenheiten und Herausforderungen zum Lernen zu schaffen, die den Kindern ,zufallen“ können. [...] Eine zentrale Aufgabe besteht darin, ,zufällige“ Lernprozesse zu ermöglichen, zu planen, vorzubereiten, zu inszenieren, zu beobachten, zu dokumentieren und für die Planung weiterer Lernanlässe auszuwerten“" (Liegle 2012, S. 17f.).

In einem weiteren spielpädagogischen Beispiel, der ebenfalls phänomenographisch meisterhaft entfalteten (und deswegen auch häufiger in der Literatur aufgegriffenen) Analyse des Bewegungsspiels „Ringelreihen“ (Brief an Cantor Carl vom 21.7.1839; Text bei Heiland 2010, S. 30f.; Hoffmann 1982) skizziert Fröbel gleichsam in nuce die Struktur seiner bildungstheoretischen Didaktik-Konzeption kategorialer Bildung, nämlich das auf die ganzheitliche Entwicklung und Förderung der Persönlichkeit hin konzentrierte Zusammenspiel von Kenntnissen, Fähigkeiten und Fertigkeiten, Gefühlen und Willensakten; transformiert in die aktuelle kompetenztheoretische Terminologie der Dimensionen von Bildungsstandards: Die Handlungskompetenz, definiert als verfügbare oder erlernbare kognitive Fähigkeiten und Fertigkeiten, verbunden mit motivationalen, volitionalen und sozialen Bereitschaften und Fähigkeiten, um konstruktiv und kooperativ variable Situationen erfolgreich und verantwortungsvoll zu bewältigen (vgl. Weinert 2001, S. 45ff.).

Fröbel demonstriert, dass insgesamt zwölf grundlegende Kompetenzen implizierende Aspekte in dem simplen Kinderlied „Ringel, Ringelreihe, es sind der Kinder dreie, steigen auf den Hollerbusch, rufen alle husch, husch, husch!“ angesprochen sind, nämlich: „Auffassung eines Kreises, einer Kreislinie“, „Auffassung des Kindes in der Mehrheit“, „Zählen“, „bestimmtes Auffassen der Kinder als Daseyende“, „ihre objektive Auffassung“, „Anschauung einer Thätigkeit zu einem Gegenstand nach dem oberen Teil desselben“, „Wahrnehmung [...] einer hörbaren Äußerung“, die ,allen“ gilt, sowie das ,ganz persönliche Sich-Wahrnehmen im Gefühle und durch die Empfindung“. Erwachsene wie Kinder „ergötzt“ das Lied immer wieder, „,weil es dem Kinde, wenn auch in seinem ganz dunkelen Gefühle, den Weg bezeichnet, wie es durch bestimmte und klare geeinte Auffassung des Äußeren, Daseyenden, des Gegebenen (Ringel, Ringelreihe) endlich zum lebhaften (angenehmen oder unangenehmen) Gefühle seiner selbst, und so zum Fühlen, zum Wahrnehmen, zum Denken seines Selbstes, seines Innersten, seines Wesens kommt. In dieser tiefen Lebensbeziehung scheint mir der ewig jugendliche Reiz dieses und jedes derartigen Liedchens und Kinderspieles für das Kind zu liegen“"(vgl. Heiland 2010, S. 30f.).

Heilands Interpretation des Textes mündet in die Feststellung, dass im analysierten Spiel „Aspekte der Wirklichkeitserfahrung und des Aufbaus von kategorialer Einsicht mit der Erfahrung der kontinuierlichen Zuwendung von Erwachsenen integriert und so freies Spiel, didaktisch-strukturelle Erfahrung und [...] personale Identität ermöglicht“" werden (Heiland 2010, S. 31). Dabei bleibt allerdings an dieser Stelle der zentral wichtige Aspekt der dem Spiel als Weltsymbol von Fröbel grundsätzlich zugeschriebenen, sinnbildlichen Funktion unerwähnt. Im Spiel als symbolischem „Vorbild und Nachbild des gesamten Menschenlebens“ (Fröbel 1982b (Bd. 2), S. 36) lernt der Mensch, „zu einer sinnigen, bedeutungsvollen Ansicht des Lebens, zur Beachtung der einzelnen Lebenserscheinungen [...] als Glieder eines großen Lebensganzen zu gelangen“ (Fröbel 1982b (Bd. 1), S. 96). Durch die sinnbildliche Anschauung ,,lernt' das Kind, der Mensch die Dinge in Beziehung zueinander und zu sich zu setzen, durch sie gewinnt die Konstruktion von Welt ,Sinn“" (Proll 1999, S. 148). 
Spielen und Lernen sind nicht einfach zwei Seiten derselben Medaille. Spielen begründet eine eigenständige Dimension der Lebenserfahrung und bedarf insofern sorgfältiger „Pflege“. „Gerade im Spielen und nur im Spielen kann das Kind und der Erwachsene sich kreativ entfalten und seine ganze Persönlichkeit einsetzen, und nur in der kreativen Entfaltung kann das Individuum sich selbst entdecken." (Winnicott 2011, S. 66) Mit der feinfühligen Übernahme der kindlichen Perspektive durch „Spielpflege“ können die Erwachsenen in mancher Hinsicht eher von den Kindern lernen als umgekehrt. „Das Spiel als spontaner Ausdruck der jeweiligen individuellen Subjektivität, Ort auch des spontanen Austauschs mit der Welt, macht auf den existentiellen, unhintergehbaren Grund des menschlichen In-der-Welt-Seins aufmerksam." (Liebau 2002, S. 19f.)

Die zentrale Aufgabe der frühpädagogischen Phase der „Spielpflege“ besteht für Fröbel in der Sicherstellung eines ursprünglichen Vertrauens durch das „erste Gefühl des Gemeinsamen, der Gemeinsamkeit" (Fröbel 1982b, (Bd. 2), S. 23), um den stetigen Aufbau einer Kultur der Verständigung zu gewährleisten, und zwar dadurch, dass die Strukturen einer humanen Weltgestaltung als Angebot gezeigt und zu Eigen gemacht werden können. Für Fröbel besteht dabei kein Zweifel, dass dieser Weg der pädagogisch arrangierten Auseinandersetzung mit Welt und Selbst als Begreifen und Gestalten des eigenen Lebens letztlich nur in einer Sprache gelingen kann, die Welt und Leben als göttliche Offenbarung, also Prozess einer religiösen Selbstverständigung und Bindung beschreibt und erklärt.

\section{Welt- und Selbsterkenntnis im Dialog mit Gott und Welt - Zur Aktualität von Fröbels Anthropologie und Bildungsphilosophie}

Während sich die Anschlussfähigkeit oder sogar eine besondere „Zukunftsfähigkeit“ des frühpädagogischen „Klassikers“ Friedrich Fröbel an die aktuelle bildungs- und kindheitstheoretische, die bildungs- und professionspolitische, die lerntheoretische, die curriculardidaktische oder die kognitionspsychologische und neurobiologische Diskussion deutlich demonstrieren lässt und in der internationalen Diskussion auch so wahrgenommen wird, ist ein essentieller Aspekt seines Werkes stark in den Hintergrund gerückt, oft schon wegen vermeintlicher „Unverständlichkeit“, nämlich der mit seiner „Sphärephilosophie“, also metaphysisch-religiös begründete Charakter seines Menschen- und damit auch Kindheitsbildes. Dieses Bild vom Kind auf der Basis einer holistisch-integralen Anthropologie bildet aber das eigentliche Zentrum seiner pädagogischen Programmatik. Während die dominierende Bildungskonzeption der Postmoderne, bezogen auf ein Weltbild kultureller Diversität, sozialer Komplexität und kulturellen Wertewandels, in einer Gesellschaft fragmentierter Lebensbereiche, metaphysischen Argumentationsformaten und erst recht holistischanthropologischen Paradigmen äußerst reserviert gegenübersteht, ist für Fröbel die „Stellung des Menschen im Kosmos“ (Scheler 1949), also die anthropologische, die religiöskosmische Einbettung der kindlich-selbstschöpferischen Tätigkeit selbstverständliche Prämisse seines frühpädagogischen Konzepts. Für ihn wäre es geradezu paradox, ein Recht des Kindes auf Religion besonders einfordern zu müssen (Bäcker-Braun/Arnold 2011; Scheilke/Schweitzer 1999). Kinder brauchen ein Bild vom Menschen im Sinne dessen, was zum Menschsein unbedingt dazugehört. In diesem Sinne war für Fröbel der Transzendenzbezug sogar eine wesentliche Voraussetzung für eine Selbstwerdung in Freiheit. 
Die Frage, ob die Dimension einer (auch) metaphysisch-religiösen Begründung des Erziehungsprozesses, von Fröbel in der sprechenden Formel der „Lebenseinigung“ zusammengefasst, bei der Sorge um das Wohl des Kindes hintangestellt werden kann, bleibt von ungebrochener Aktualität. Wenn bis weit ins 20. Jahrhundert hinein „die Genese der Pädagogik aus dem Geist der Theologie“ (Tenorth 2000) unbestritten war, z.B. noch in der Reformpädagogik die Transformationen des Religiösen zu einer besonders engen Verbindung von theologisch-religiösen und pädagogischen Konzepten führten (vgl. Baader 2005), hat die Erziehungswissenschaft seit Ende der 1960er Jahre „das generelle Thema der Religion aus den Augen verloren“ (Nipkow 2010, S. 822). Der Rückblick auf Fröbels Bildungstheorie heute lässt einmal mehr deutlich werden, dass die Erziehungswissenschaft ,bisher noch keine bildungstheoretisch bedeutsame, neue Debatte über das Verhältnis von Religion und Bildung hervorgebracht“ hat (Benner/Tenorth 1996, S. 3ff.), trotz des gelegentlich betonten Hinweises, dass Religion nicht ausschließlich „Privatsache“ sei. „Es geht die Gemeinschaft sehr wohl an, ob da etwas ist, was Verständigung verhindert oder für überflüssig erklärt oder ihr den richtigen Erkenntnisgrund anweist, einen der nicht in der natürlichen Neugier, in der Beherrschung der Natur, in der bestmöglichen Nutzung der Lebenszeit und der Welt aufgeht.“ (von Hentig 1992, S. 24f.)

Fröbels spielpädagogische Bildungstheorie bringt eindrücklich in Erinnerung, dass es bei weitem nicht ausreichen könnte, im Kontext standardorientierter Bildungspläne oder Curricula religiöse Kompetenz zwar aufzunehmen, ihr faktisch aber eine neben- oder nachgeordnete Randzone zuzuweisen. Vielmehr sollte „es ein unverzichtbares Qualitätsmerkmal aller Kindertagesstätten sein, dass Religion in ihnen vorkommt" (Harz 1999, S. 63). Jürgen Habermas hat in seiner Dankesrede bei der Verleihung des Friedenspreises des Deutschen Buchhandels 2001 nachdrücklich auf Religion als Ressource für die moderne Gesellschaft hingewiesen, um der „schleichenden Entropie der knappen Ressource Sinn“ entgegenzuwirken, den Verlust zu begreifen und zu vermeiden, den das fortschreitende Abhandenkommen der Verstehensmechanismen für Religion bedeutet (vgl. Horster 2006, S. 41ff.).

Fröbel kombinierte in seiner sphärephilosophisch begründeten Anthropologie als der Grundlage seines Modells, die Welt als pädagogische Realität zu gestalten, Elemente der zeitgenössischen Identitätsphilosophie, christlichen Theologie und Naturwissenschaft bzw. Mathematik. Ich möchte die Einschätzung wagen, dass Fröbel heute mit Enthusiasmus zugreifen würde auf den Forschungsfundus der Lebenswissenschaften, vor allem auch die Evolutionstheorie, insbesondere auch deren Ausstrahlung in die Philosophie, z.B. das von Wolfgang Welsch, ,jenseits der anthropischen Denkform der Moderne“, konstruierte Modell vom „Homo mundanus“ (2012a). Dies ließe sich in seinen Basisannahmen durchaus als aktuelle Version des Fröbelschen Zentralkonzepts des „Entgegengesetztgleichen“ von Geist und Natur lesen und auf seine pädagogisch-anthropologischen Konsequenzen hin explizieren, also auch für eine aktuelle Perspektive des Verhältnisses von Menschenbild und Bildung. Welschs „konsequent evolutionistische Perspektive“ für die Anthropologie, Ontologie und Epistemologie weist insofern eine weiterführende strukturelle Analogie zu Fröbels identitätstheoretischer Sphärephilosophie auf, als beide von einer „elementaren Weltverbundenheit des Menschen“ (Welsch 2012b) als anthropologischer Grundgegebenheit ausgehen.

Fröbel ist im Zuge seiner lebenslangen Bemühungen, „Menschenerziehung“ theoretisch zu modellieren und praktisch zu implementieren, immer wieder auf die kulturelle Vielfalt des Kinderspiels als „Schlüssel“ zur Welt gestoßen. Seine in ihrer phänomeno- 
graphischen Präzision kaum übertreffbaren Deskriptionen von Kinderspielen bzw. Spielsituationen, in der Forschungsliteratur immer wieder als „genial“ bezeichnet, eröffnen heute wohl am ehesten einen direkten Zugang zu Fröbels Texten. Fröbels Faszination am Phänomen „Kinderlied“ beispielsweise dürfte ihren tieferen Sinn darin begründet finden, dass und in welcher Gestalt unser Welt-Verstehen hier einen sprechenden Ausdruck dafür findet, wie - und dafür stehen die Kinder geradezu exemplarisch - in Spiel, Lied und Sprache sich ein Blick auf, eine Möglichkeit der Erfahrung vom Sinn des Ganzen eröffnet, so auch die Relevanz der religiösen Welt-Perspektive zeigt, in einem weiteren Schritt auch die der religiösen Bindung, wie sie für Fröbel konstitutives Element der Begründung des kindlichen Bildungsprozesses darstellt.

Die „Ahnung“ von und schließlich die bewusste, lebenslang aufrecht erhaltene Suche des Menschen nach dem Sinn des Ganzen, bis hin zur Frage, worin der Sinn alles dessen besteht, von dem wir meinen oder sogar überzeugt sind und glauben, dass es als gesichertes Wissen sinnvoll ist, kann man schwerlich als das individuell-private Problem eines gläubigen Menschen - in Fröbels Fall eines gläubigen Christen - einordnen und damit auch als schon erledigt ansehen, vielleicht gerade einmal achselzuckend als „Kinderfrage“ gelten lassen. Da die hier thematisierte Dimension einer metaphysisch-religiösen WeltDeutung an dieser Stelle nicht systematisch entfaltet oder gar diskutiert werden kann, sollen für eine grundlegend andere Sichtweise stellvertretend zwei Philosophen zu Wort kommen, die gegenwärtig zu den renommiertesten ihrer Zunft in Deutschland zählen: Volker Gerhardt und Peter Sloterdijk. Beide entwickeln, wie Fröbel, ihre Grundthesen über das Verhältnis von Mensch und Welt gelegentlich auch im Rückgriff auf ein Kinderlied bzw. ein Kinderspiel.

In seinem Werk „Vom Sinn des Sinns“ (2015), im letzten Jahr erschienen, mittlerweile schon in dritter Auflage, geht Gerhardt auf die Suche nach dem Ort des „Göttlichen“ zwischen Denken, Wissen und Glauben, danach, wie ein Mensch sich als solcher, als Person begreifen kann. Im Kern geht es um die begriffliche Kennzeichnung der Relation zwischen Welt und Individuum. „Es ist das Ganze der Welt, der gegenüber das Ganze eines Individuums zu seiner eigenartigen Bedeutung gelangt. Wem diese Steigerung im ausgehaltenen Gegensatz zu dem ihm prinzipiell Gleichen zu hoch erscheint, der denke nur an das [...] Kinderlied, in dem die Relation zwischen Welt und Individuum offenbar schon von den Kleinsten in seiner unendlichen Bedeutung erfasst werden kann. Bis heute verspüre ich den abenteuerlichen Schauer, den mir die verlockende Vorstellung erzeugte, ich könnte selbst ,mit Stock und Hut' , in die weite Welt hinein' spazieren. Das Kind geht nicht hinaus, sondern hinein - ins Ganze, zu dem es gehört. Es hält das Ganze aus, und es wächst dadurch, dass es sich ihm in der eigenen Tätigkeit aussetzt. Deshalb ist es allemal zu wenig, die Welt und Selbst umfassende Einheit nach Maßgabe eines lediglich von außen erkennbaren Gegenstands zu begreifen. Sie muss vielmehr auch die Eigenschaft haben, die den handelnden, beobachtenden und erkennenden Menschen dazu bringen kann, sich dieser Einheit des Ganzen zuzurechnen." Hier, das ist Gerhardts Folgerung, ist - und das in langer philosophisch-religiöser Tradition - der Gottesbegriff anzusiedeln, durchaus als ein Produkt der Erkenntnis, nicht allein des Glaubens. Dem Einen des Ganzen wird ein Name gegeben, „der dem Ganzen korrespondiert, das sich als Teil der Welt und dennoch als deren Gegenüber begreift. Eine solche Relation gibt es nur unter den Bedingungen der Erkenntnis, in der man sich beispielsweise als Teil einer Familie und dennoch als Einheit verstehen kann, dem die Familie fremd werden kann. Es ist der Vorzug des Gottesbegriffs, dieses Ganze des Daseins in umfänglichster Weise zum Ausdruck zu bringen und 
darin zugleich ein inneres Verhältnis kenntlich zu machen, das durch mehr oder weniger Nähe gekennzeichnet sein kann. [...] In Gott also wäre alles aufgehoben, einschließlich des die Welt wie von außen erkennenden Selbst. Der Begriff Gottes erlaubt die alles einbeziehende Einheit in Verbindung mit der Selbstständigkeit des Denkenden zu denken“ (Gerhardt 2015, S. 46f.).

In einem solchen Kontext metaphysischer Analyse der Welt-Mensch-Relation erhalten die Fröbelschen Leitbegriffe kategorialer Bildung wie „Einheit“, „Allheit“, „Gegensätzlichkeit“, die, wie in Abschnitt 2 dargelegt, für gegenwärtig dominierende Ansätze zur Modellierung von Bildungsprozessen geradezu wie aus der Zeit gefallen erschienen, durchaus die ihnen von Fröbel zugedachte Bedeutsamkeit. Sie sind „Schlüssel“, „Lebenseinigung“ einsichtig zu machen.

Das zweite Beispiel „Wo sind wir, wenn wir in der Welt sind?“ stammt aus Peter Sloterdijks voluminöser Suche nach der Genese und den Varianten der Bedeutung des Begriffs „Sphäre“ (Sloterdijk 1999), dabei zunächst dessen Verwendung bei der Beschreibung der Stellung des Menschen im Kosmos - damit eine Thematik aufnehmend, die einen Fröbel-Interessierten, der versucht, in das Arkanum von Fröbels Sphärephilosophie begrifflich vorzudringen, geradezu neugierig machen muss. Zwei Aspekte sollen bei dem Vorhaben, Fröbel und Sloterdijk in einem doch kühnen Vergleich in Beziehung zu setzen, herausgestellt werden: Es ist zum einen - ähnlich wie bei Fröbel - die Brillanz einer eindringlichen phänomenographischen Deskription eines Kinderspiels, das Beispiel des „kleinen Seifenblasenkünstlers auf dem Balkon“; zum anderen wird aus dieser empirischen Deskription analytisch-systematisch das Designat eines gehaltvollen kosmologischen Begriffs von „Sphäre“, abgegrenzt gegenüber seinen traditionell religiösen Konnotationen, entwickelt. Da die Validität der in dichter Beschreibung erzeugten Aussagen des Autors zum ablaufenden Geschehen nur auf dem Wege der Evidenzerfahrung im Nachvollzug der Interpretamente geprüft werden kann, erscheint eine ausführlichere Zitation unumgänglich:

„Das beschenkte Kind steht fiebernd auf dem Balkon und schaut den Seifenblasen nach, die es aus der kleinen Schlaufe vor seinem Mund in den Himmel bläst. Jetzt sprudelt ein Bläschenschwarm in die Höhe, chaotisch munter wie ein Wurf von blauschimmernden Murmeln. Dann, bei einem nächsten Versuch, löst sich zitternd, von einem ängstlichen Leben gefüllt, ein großer ovaler Ballon von der Schlaufe, [...] schwebt vorwärts hinunter auf die Straße. Ihm folgt die Hoffnung des entzückten Kindes. Dieses schwebt selbst mit seiner Wunderblase in den Raum hinaus, als hinge für Sekunden sein Schicksal an dem nervösen Gebilde. Wenn die Blase endlich nach bebendem, gedehntem Flug zerplatzt, gibt der Seifenblasenkünstler auf dem Balkon einen Laut von sich, der zugleich ein Seufzer ist und ein Jubelruf. Für die Lebensspanne der Blase war der Bläser außer sich gewesen, als habe der Bestand der Kugel davon abgehangen, daß sie in eine mithinausschwingende Aufmerksamkeit eingehüllt blieb. [...] Die Aufmerksamkeit des kleinen Zauberers fliegt auf ihrer Spur ins Weite und stützt die dünnen Wände der gehauchten Körper mit ihrem begeisterten Dabeisein. Zwischen der Seifenblase und ihrem Bläser herrscht eine Solidarität, die den Rest der Welt ausschließt. [...] In den Kugeln hat sich sein Exhalat von ihm losgelöst und wird von der Brise bewahrt und weitergetragen; zugleich ist das Kind von sich selbst entrückt, indem es sich verliert in das atemlose Mitfliegen seiner Aufmerksamkeit durch den beseelten Raum. [...] Das Kind, das seinen Seifenblasen ins Offene folgt, ist kein cartesisches Subjekt, das in seinem ausdehnungslosen Denk-Punkt verharrt, während es ein ausgedehntes Ding auf seiner Bahn durch den Raum beobachtet. Begeistert solidarisch mit seinen schillernden Kugeln stürzt sich der experimentierende Spieler in den offenen Raum und verwandelt die Zone zwischen Auge und Gegenstand in eine beseelte Sphäre. [...] Unmerklich geht so dem Spielenden inmitten seiner glücklichen Unterhaltung eine Einsicht auf, die er unter schulischen Mühen später wieder verlernen wird: daß der Geist auf seine Weise selbst im Raum ist. [...] Tatsächlich ist das Bedürfnis bekannt - Schopenhauer hat es das metaphysische genannt -, daß 
alles, was der Welt oder dem Seienden im ganzen angehört, in einem Hauch wie in einem untilgbaren Sinn enthalten sein möge. [...] Auf die gnostisch inspirierte Frage: Wo sind wir, wenn wir in der Welt sind? ist eine kompetente zeitgenössische Antwort möglich. Wir sind in einem Außen, das Innenwelten trägt. Mit der These vom Prius des Außen vor Augen brauchen wir keine naiven Nachforschungen über die Stellung des Menschen im Kosmos mehr anzustellen. [...] Darum ist die Erkundigung nach unserem Wo sinnvoller denn je, denn sie richtet sich auf den Ort, den Menschen erzeugen, um zu haben, worin sie vorkommen können als die, die sie sind. Dieser Ort trägt hier, einer altehrwürdigen Überlieferung eingedenk, den Namen S p h ä r e. Die Sphäre ist das innenhafte, erschlossene, geteilte Runde, das Menschen bewohnen, sofern es ihnen gelingt, Menschen zu werden. [...] In Sphären leben heißt, die Dimension erzeugen, in der Menschen enthalten sein können." (Sloterdiyk 1999, S. 17ff.)

In seiner phänomenologischen Existenzhermeneutik entwirft Sloterdijk ein anthropologisches Konzept von Menschsein als Wohnen in der Welt, gleichsam ein säkularisiertes Modell von „Sphäre“, jenseits traditioneller Religiosität, aber ohne eine religiösmetaphysische Intention als Grundbedürfnis auch wieder nicht denkbar, wenn der Mensch seinen Ort als Mensch in der Welt finden will.

Anhand der beiden zeitgenössischen Beispiele philosophischer Konstrukte zum Stellenwert der Bedeutung eines religiös-metaphysisch begründeten Selbstverständnisses des Menschen in der Moderne ist zu hoffen, dass immerhin ein Zugang zur Auflösung der Problematik eröffnet werden konnte, warum Fröbel der religiösen Dimension menschlicher Existenz durchgängig eine Basisfunktion in seinem Konzept von Menschenerziehung zugemessen hat. Nicht zuletzt die ebenso präzise wie einfühlsam-liebevolle Beobachtung der kleinen Kinder in seinen letzten beiden Lebensjahrzehnten hat ihn in der Festlegung der anthropologischen Prämissen seines Menschenbildes, durchaus also auch empirisch bewährt, bestärkt. Mit seinem Konzept vom Kindergarten als einem die „,sphärische“ Grundverfasstheit der Welt repräsentierenden Ort für humanes „Wohnen“ in der Welt hat er offenbar ein kontinuierlich weiterwirkendes „klassisches“ Modell des Lebensortes für Kinder in ihrer sie jeweils umgebenden Gesellschaft nicht nur begründet, sondern auch schon experimentell erfolgreich realisiert. Kann ein pädagogisch-didaktisches Konzept von Menschenbildung, ausgerichtet am Leitbild der Autonomie schon des Kindes als Subjekt, - zugegeben salopp formuliert - „moderner“ im Sinne von ,aktueller“ sein, wenn es wirklich um die zentrale Frage nach dem Erwerb von „Lebenskompetenz“ geht, also die Bedingungen der Ermöglichung humaner Existenz über nachweislichen optimalen beruflich-gesellschaftlichen und ökonomischen Erfolg hinaus?

Fröbelexperten wie Helmut Heiland, Ludwig Liegle oder Michael Winkler haben in unterschiedlicher Weise darauf hingewiesen, dass der Reichtum der Gedankenwelt Fröbels bei weitem noch nicht ausgeschöpft, vielleicht noch nicht einmal richtig erkannt wurde, wie „modern“ oder, wie eingangs beschrieben, „zukunftsfähig“ Friedrich Fröbel in seinem Werk und als Person ist.

\section{Literatur}

Ahnert, L. (2010): Wieviel Mutter braucht ein Kind? Bindung - Bildung - Betreuung. Öffentlich und privat. - Heidelberg.

Andresen, S. (2012): Was unsere Kinder glücklich macht. Lebenswelten von Kindern verstehen. - Freiburg.

Baader, M. S. (2005): Erziehung als Erlösung. Transformationen des Religiösen in der Reformpädagogik. - Weinheim, München. 
Bäcker-Braun, K./Arnold, M. (2011): Religiöses Erleben von Anfang an. Rituale, Spiele und Lieder für Krippe, Kita und Eltern-Kind-Gruppen. - München.

Benner, D./Tenorth, H.-E. (1996): Bildung zwischen Staat und Gesellschaft. Zeitschrift für Pädagogik, 42, S. 3-14.

Bucher, A./Münnich, S. (2012): Was bedeutet Glück? Glücksgefühle bei Kindern und Pädagogen. Klein \& groß, Lebensorte für Kinder, 65, 4, S. 14-15.

Bundesministerium für Familien, Senioren, Frauen und Jugend (BMFSFJ) (Hrsg.) (1998): Zehnter Kinder- und Jugendbericht. - Bonn, Berlin.

Bundesministerium für Familien, Senioren, Frauen und Jugend (BMFSFJ) (Hrsg.) (2005): Zwölfter Kinder- und Jugendbericht. - Berlin.

Deutsches PISA-Konsortium (Hrsg.) (2001): PISA 2000. Basiskompetenzen von Schülerinnen und Schülern im internationalen Vergleich. - Opladen.

Drieschner, E. (2011): Bindung in familialer und öffentlicher Erziehung. Zum Zusammenhang von psychischer Sicherheit, Explorationssicherheit und früher Bildung im geteilten Betreuungsfeld. In: Drieschner, E./Gaus, D. (Hrsg.): Liebe in Zeiten pädagogischer Professionalisierung. - Wiesbaden, S. $105-156$.

Ebert, S. (Hrsg.) (1991): Zukunft für Kinder. Grundlagen einer übergreifenden Politik. - München.

Erenz, B. (Hrsg.) (2009): Vordenker, Vorbilder, Visionäre. 50 Denker von gestern für die Welt von morgen. ZEIT - Geschichte, Nr. 74, 64.

Erning, G./Neumann, K./Reyer, J. (Hrsg.) (1987): Geschichte des Kindergartens. 2 Bände. - Freiburg .

Fröbel, F. (1982a): „Kommt, lasst uns unseren Kindern leben“! Aus dem pädagogischen Werk eines Menschenerziehers, hgg. von Boldt, R./Knechtel, E./König, H., Berliner Ausgabe, 3 Bände. - Berlin.

Fröbel, F. (1982b): Ausgewählte Schriften, hgg. von Hoffmann, E./Heiland, H./Wächter, R., Stuttgarter Ausgabe, 5 Bände. - Stuttgart.

Fthenakis, W. E. (Hrsg.) (2003): Elementarpädagogik nach PISA. - Freiburg.

Gerhardt, V. (2015): Der Sinn des Sinns. Versuch über das Göttliche. - München.

Harz, F. (1999): Die religiöse Dimension bei der qualitativen Profilierung evangelischer Tageseinrichtungen für Kinder. In: Bremische Evangelische Kirche, Landesverband Ev. Tageseinrichtungen für Kinder (Hrsg.): Qualität für Kinder zwischen Markt und Menschlichkeit. - Seelze, S. 61-76.

Hasselhorn, M. (2005): Lernen im Altersbereich zwischen 4 und 8 Jahren. In: Guldimann, T./Hauser, B. (Hrsg.): Bildung 4- bis 8-jähriger Kinder. - Münster, S. 77-88.

Hebenstreit, S. (2003): Friedrich Fröbel - Menschenbild, Kindergartenpädagogik, Spielförderung. - Jena.

Heiland, H. (2010): Fröbels Pädagogik der Kindheit - Didaktische Überlegungen zur Spielpädagogik. In: Kasüschke, D. (Hrsg.): Didaktik in der Pädagogik der frühen Kindheit. - Köln, Kronach, S. 15-44.

Heiland, H./Neumann, K. (Hrsg.) (1998): Friedrich Fröbel in internationaler Perspektive. Fröbelforschung in Japan und Deutschland. - Weinheim.

Heiland, H./Neumann, K. (Hrsg.) (2003): Fröbels Pädagogik verstehen, interpretieren, weiterführen. Internationale Ergebnisse zur neueren Fröbelforschung. - Würzburg.

Heiland, H./Neumann, K./Gebel, M. (Hrsg.) (1999): Friedrich Fröbel. Aspekte international vergleichender Historiographie. - Weinheim.

Heller, E. (2013): Im Spiel die Welt begreifen. - Berlin.

Hoffmann, E. (1982): Ein unveröffentlichter Fröbel-Brief über die Bildung der Kinder. Zeitschrift für Pädagogik, 28, S. 175-192.

Horster, D. (2006): Jürgen Habermas und der Papst. Glauben und Vernunft, Gerechtigkeit und Nächstenliebe im säkularen Staat. - Bielefeld.

Jugendministerkonferenz/Kultusministerkonferenz (JMK/KMK) (2004): Gemeinsamer Rahmen der Länder für die frühe Bildung in Kindertageseinrichtungen. Beschluss vom 13./14.5.2004. - Berlin.

Kasüschke, D. (Hrsg.) (2010): Didaktik in der Pädagogik der frühen Kindheit. - Köln, Kronach.

Kerstan, Th. (2009): Friedrich Fröbel. In: Erenz, B. (Hrsg.): Vordenker, Vorbilder, Visionäre. 50 Denker von gestern für die Welt von morgen. ZEIT - Geschichte, Nr. 74, 64, S. 15.

Klafki, W. (1963): Das pädagogische Problem des Elementaren und die Theorie der Kategorialen Bildung. - Weinheim.

Klieme, E./Avenarius, H./Blum, W./Döbrich, P./Gruber, H./Prenzel, M. u.a. (2003): Zur Entwicklung nationaler Bildungsstandards: Eine Expertise. - Berlin. 
Köller, O. (2008): Bildungsstandards - Verfahren und Kriterien bei der Entwicklung von Messinstrumenten. Zeitschrift für Pädagogik, 54, 2, S. 163-173.

Krause, S./Nikolova, R./Schluß, H./Weiß, T./Willems, J. (2008): Kompetenzerwerb im evangelischen Religionsunterricht. Ergebnisse der Konstruktvalidierungsstudie der DFG-Projekte RU-Bi-Qua/KERK. Zeitschrift für Pädagogik, 54, 2, S. 174-188.

Liebau, E. (2002): Jugendhilfe, Bildung, Teilhabe. Bildung als Teilhabebefähigung. In: Münchmeier, R./Otto, H.-U./Rabe-Kleberg, U. (Hrsg.): Bildung und Lebenskompetenz. Kinder und Jugendhilfe vor neuen Aufgaben. - Opladen, S. 19-27.

Liegle, L. (2012): Perspektiven einer frühpädagogischen Didaktik der indirekten Erziehung. Fröbel revisited. In: Neumann, K./Sauerbrey, U./ Winkler, M. (Hrsg.): Fröbelpädagogik im Kontext der Praxis. - Bad Berka, S. 10-20.

Liegle, L. (2013): Frühpädagogik. Erziehung und Bildung kleiner Kinder. Ein dialogischer Ansatz. Stuttgart.

Meyerhöfer, W. (2010): Welche Mathematik gehört in den Kindergarten? In: Neumann, K./Sauerbrey, U./Winkler, M. (Hrsg.): Fröbelpädagogik im Kontext der Moderne. - Jena, S. 235-251.

Münchmeier, R./Otto, H.-U./Rabe-Kleberg, U. (Hrsg.) (2002): Bildung und Lebenskompetenz. Kinder und Jugendhilfe vor neuen Aufgaben. - Opladen.

Neumann, K. (1993): Zum Wandel der Kindheit vom Ausgang des Mittelalters bis an die Schwelle des 20. Jahrhunderts. In: Markefka, M./Nauck, B. (Hrsg.): Handbuch der Kindheitsforschung. - Neuwied, Kriftel, Berlin, S. 191-205.

Neumann, K. (2013a): Kindheitsbilder - im Perspektivengeflecht von Mythen, Expertenwissen und Lebenswelt. In: Förster, Ch./Höhn, K./Schreiner, S. A. (Hrsg.): Kindheitsbilder - Familienrealitäten. Freiburg, Basel ,Wien, S. 14-24.

Neumann, K. (2013b): Klassiker der Pädagogik der frühen Kindheit. In: Fried, L./Roux, S. (Hrsg.): Handbuch Pädagogik der frühen Kindheit. - Berlin, S. 107-118.

Nipkow, K. E. (2010): Religion/religiöse Erziehung. In: Benner, D./Oelkers, J. (Hrsg.): Historisches Wörterbuch der Pädagogik. - Weinheim, Basel, S. 807-823.

Pramling Samuelsson, I./Asplund Carlsson, M. (2007): Spielend lernen. Stärkung lernmethodischer Kompetenzen. - Troisdorf.

Proll, H. (1999): Der Spielbegriff Piagets und Fröbels. Spiel in der vorschulpädagogischen Diskussion. In: Heiland, H./Neumann, K./Gebel, M. (Hrsg.): Friedrich Fröbel. Aspekte international vergleichender Historiographie. - Weinheim, S. 120-151.

Reyer, J. (1988): Kindergartenprinzip und Anstaltsform. Neue Sammlung, 28, S. 486-504.

Reyer, J. (2010): Kindergarten. In: Benner, D./Oelkers, J. (Hrsg.): Historisches Wörterbuch der Pädagogik. - Weinheim, Basel, S. 518-526.

Rockstein, M. (Hrsg.) (2015): Fröbels Kindergarten. Ein Zukunftsmodell aus der Vergangenheit. - Bad Blankenburg.

Rothgangel, M. (2008): Bildungsstandards für den Religionsunterricht. Zur fachdidaktischen Konsistenz des Berliner Forschungsprojekts. Zeitschrift für Pädagogik, 54, 2, S. 194-197.

Schäfer, G. E. (2007) (Hrsg.): Bildung beginnt mit der Geburt. Ein offener Bildungsplan für Kindertageseinrichtungen in Nordrhein-Westfalen. - Berlin.

Schäfer, G. E. (2008): Das Denken lernen. Betrifft Kinder, S. 7-15.

Scheilke, Ch./Schweitzer, F. (Hrsg.) (1999): Kinder brauchen Hoffnung. Religion im Alltag des Kindergartens. Band 1. - Gütersloh.

Scheler, M. (1949): Die Stellung des Menschen im Kosmos. - München.

Schlömerkemper, J. (Hrsg.) (2004): Bildung und Standards. Zur Kritik der „Instandardsetzung“ des deutschen Bildungswesens. Die Deutsche Schule. 8. Beiheft. - Weinheim.

Sloterdijk, P. (1999): Sphären. Band 1: Blasen. - Frankfurt.

Surall, F. (2005): Kindergarten und Kinderrechte. Zum 150. Geburtstag von Kate Douglas Wiggin - einer Pionierin der Kinderrechtsbewegung. Frühe Kindheit, 8, S. 40-50.

Tenorth, H. E. (2000): Natur als Argument in der Pädagogik des 20. Jahrhunderts. In: Baader, M. S./Jacobi, J./Andresen, S. (Hrsg.): Ellen Keys reformpädagogische Vision. „Das Jahrhundert des Kindes" und seine Wirkung. - Weinheim, S. 301-322.

Tenorth, H.-E. (2008): Bildungsstandards außerhalb der „Kernfächer“. Herausforderungen für den Unterricht und die fachdidaktische Forschung. Zur Einleitung in den Thementeil. Zeitschrift für Pädagogik, 54, 2, S. 159-162. 
Tomasello, M. (2011): Die Ursprünge der menschlichen Kommunikation. - Frankfurt.

Viernickel, S./Stenger, U. (2010): Didaktische Schlüssel in der Arbeit mit null- bis dreijährigen Kindern. In: Kasüschke, D. (Hrsg.): Didaktik in der Pädagogik der frühen Kindheit. - Köln, Kronach, S. 175224.

von Hentig, H. (1992): Glaube. Fluchten aus der Aufklärung. - Düsseldorf.

Weinert, F. E. (2001): Concept of competence: A conceptual clarification. In: Rychen, D./Salganik, L.

(Hrsg.): Defining and selecting key competencies. - Kirkland, S. 45-65.

Welsch, W. (2012a): Homo mundanus. Jenseits der anthropischen Denkform der Moderne. - Weilerswirt.

Welsch, W. (2012b): Mensch und Welt. Eine evolutionäre Perspektive der Philosophie. - München.

Wigger, L. (2010): Didaktik. In: Benner, D./Oelkers, J. (Hrsg.): Historisches Wörterbuch der Pädagogik. - Weinheim, Basel, S. 245-278.

Wiggin, K. D. (1892): Children's Rights. A book of nursery logic. - Boston.

Winkler, M. (2009): Menschenerziehung - Lernen das Leben zu lesen. In: Rockstein, M./Neumann, K. (Hrsg.): Fröbels Erbe. - Bad Blankenburg, S. 57-87.

Winkler, M. (2010): Der politische und sozialpädagogische Fröbel. In: Neumann, K./Sauerbrey, U./Winkler, M. (Hrsg.): Fröbelpädagogik im Kontext der Moderne. - Jena, S. 27-51.

Winnicott, $D$. W. (2011): Vom Spiel zur Kreativität. 12. Auflage. - Stuttgart.

World Vision Deutschland (Hrsg.) (2010): Kinder in Deutschland 2010: 2. World Vision Kinderstudie. Frankfurt.

Zimmer, J. (1991): Eine Politik für Kinder als konkrete Utopie. In: Ebert, S. (Hrsg.): Zukunft für Kinder. - München, S. 237-252. 